\title{
Percepção dos idosos institucionalizados acerca da qualidade de vida
}

\author{
Perception of institutionalized elderly about quality of life
}

\author{
Percepción de ancianos institucionalizados sobre la calidad de vida
}

\begin{abstract}
Francisco Jânio Marinho de Souza ${ }^{1}$, Carmelita Maria Silva Sousa ${ }^{1}$, Allex Alves Sobral de Sousa ${ }^{1 *}$, Lucineide Coqueiro Gurgel ${ }^{1}$, Cícera Leice Silva Marques ${ }^{1}$, Janaina Brito Alves ${ }^{1}$, Maria Aparecida Feitosa Candido Herculano ${ }^{1}$, Eulina Alves Sousa Brito ${ }^{1}$, Willma José de Santana ${ }^{2}$, Dayse Christina Rodrigues Pereira Luz².
\end{abstract}

\section{RESUMO}

Objetivo: A pesquisa em foco teve como objetivo avaliar os aspectos relacionados com a qualidade de vida dos idosos institucionalizados na cidade do Crato no estado do Ceará. Métodos: O presente estudo teve caráter exploratório, descritivo e abordagem qualitativa. Traçou-se o perfil sócio demográfico e cultural; identificou-se a importância dos profissionais das Instituições de Longa Permanência para a pessoa Idosa (ILPI) e, a percepção à cerca da qualidade de vida em uma instituição. Os dados foram coletados através de uma entrevista semiestruturada, onde abordou-se o envelhecimento associado à qualidade de vida na instituição. Resultados: A amostra para o estudo contou com 13 idosos, todas do sexo feminino e com idade entre 70 e 80 anos. Foram organizadas em quatro categorias: 1) alterações morfofuncionais; 2) participação em eventos de lazer; 3) assistência profissional; e 4) qualidade de vida na ILPI. O processo de envelhecimento influencia no desenvolvimento de alterações que podem impactar de maneira importante na qualidade de vida dos idosos. Conclusão: Compreendeu-se que, mesmo frente a determinadas limitações causadas pelo aumento dos anos, as pesquisadas percebem a qualidade de vida na ILPI de forma positiva em todos os aspectos, que começou com a acolhida e continua no cotidiano da instituição.

Palavras-chave: Idoso, Instituição, Qualidade de vida.

\begin{abstract}
Objective: This research aimed to evaluate aspects related to the quality of life of institutionalized elderly in the city of Crato in the state of Ceará. Methods: This study was exploratory, descriptive and qualitative approach. The socio-demographic and cultural profile was traced; We identified the importance of the professionals of the Long Stay Institutions for the Elderly (LSIE) and the perception about the quality of life in an institution. Data were collected through a semi-structured interview, which addressed the aging associated with quality of life in the institution. Results: The sample for the study consisted of 13 elderlies, all female and aged between 70 and 80 years. They were organized into four categories: 1) morphofunctional changes; 2) participation in leisure events; 3) professional assistance; and 4) quality of life in LSIE. The aging process influences the development of changes that can have an important impact on the quality of life of the elderly. Conclusion: It was understood that, even in the face of certain limitations caused by the increase of years, the respondents perceive the quality of life in LSIE in a positive way in all aspects, which began with the welcome and continues in the daily life of the institution.
\end{abstract}

Keywords: Elderly, Institution, Quality of life.

\footnotetext{
${ }^{1}$ Mestrando da Atenas College University. Orlando-Flórida-USA. *E-mail: allexsobralfisio@hotmail.com

${ }^{2}$ Faculdade do Juazeiro do Norte (FJN). Juazeiro do Norte, CE.
} 


\section{RESUMEN}

Objetivo: Esta investigación tuvo como objetivo evaluar aspectos relacionados con la calidad de vida de ancianos institucionalizados en la ciudad de Crato en el estado de Ceará. Métodos: Este estudio fue exploratorio, descriptivo y cualitativo. Se trazó el perfil sociodemográfico y cultural; Identificamos la importancia de los profesionales de las instituciones de larga estadía para personas mayores (LSIE) y la percepción sobre la calidad de vida en una institución. Los datos fueron recolectados a través de una entrevista semiestructurada, que abordó el envejecimiento asociado con la calidad de vida en la institución. Resultados: La muestra para el estudio consistió en 13 ancianos, todos mujeres y de edades comprendidas entre 70 y 80 años. Se organizaron en cuatro categorías: 1) cambios morfofuncionales; 2) participación en eventos de ocio; 3) asistencia profesional; y 4) calidad de vida en LSIE. El proceso de envejecimiento influye en el desarrollo de cambios que pueden tener un impacto importante en la calidad de vida de los ancianos. Conclusion: Se entendió que, incluso ante ciertas limitaciones causadas por el aumento de los años, los encuestados perciben la calidad de vida en LSIE de manera positiva en todos los aspectos, lo que comenzó con la bienvenida y continúa en la vida diaria de la institución.

Palabras clave: Ancianos, Institución, Calidad de vida.

\section{INTRODUÇÃO}

A Organização Mundial de Saúde (OMS) considera idosa pessoa com idade a partir de 60 anos, porém essa consideração diz respeito exclusivamente ao envelhecimento biológico o que não impede de a pessoa ser socialmente e intelectualmente ativa (OMS, 2016). À medida que, a população envelhece faz-se necessário um novo olhar no sentido de proporcionar uma melhor qualidade de vida em qualquer espaço de inserção (ABREU JF, et al., 2017).

Ressalta-se que com o envelhecimento surgem as mudanças morfofuncionais que incide na qualidade de vida dos idosos, incapacitando-os de realizar atividades do cotidiano. O processo de envelhecimento é considerado dinâmico, gradativo, e recheado de alterações físicas e psicossociais, emocionais ou cognitivas, assim essas mudanças tornam os idosos mais susceptíveis e vulneráveis a certos agravos na saúde, assim como o aparecimento de patologias e que incidem diretamente sobre os idosos o que prejudica a qualidade de vida (OLIVEIRA TC, et al., 2015).

A degradação progressiva de órgãos e suas respectivas funções é algo comum de ocorrer conforme a idade avança. É necessário considerar esses fatores em uma abordagem conceitual de qualidade de vida e possíveis fatores que podem interferir no desenvolvimento do processo. Considerando esse conceito, tem-se ainda o envelhecimento patológico, em que a partir de doenças de base podem prejudicar a saúde do indivíduo de uma maneira importante e assim também prejudicando de maneira significativa a qualidade de vida dessa população (KHAURY HTT e NEVES ACS, 2014).

A limitação progressiva dos órgãos e suas respectivas funções tornam-se comum no envelhecimento. Assim, faz-se necessário considerar essa mudança em uma abordagem conceitual sobre qualidade de vida e os fatores que interferem nesse processo. Percebe-se que, a qualidade de vida tem sido uma preocupação do ser humano na sociedade atual. Em se tratando da pessoa idosa em envelhecimento torna-se até um compromisso uma vez que, é direito envelhecer com qualidade de vida psicossocial (KLEIN LH e LODOVICI FMM, 2015).

A qualidade de vida, portanto, tem sido preocupação constante do ser humano desde o início de sua existência, uma vez que envelhecer de modo saudável se constitui num compromisso pessoal na busca contínua de uma vida desenvolvida à luz de um bem-estar indissociável das condições do modo de viver, que está intimamente ligado à saúde, moradia, educação, lazer, transporte, liberdade, trabalho, autoestima, entre outros (SANTOS FH, et al., 2014).

O aumento da participação da pessoa idosa na sociedade fomenta a necessidade de atendimento a essa demanda e, a criação de vagas em determinados serviços públicos como os de acompanhamento, abrigo e saúde. As Instituições de Longa Permanência para a pessoa idosa (ILPIs) são consideradas atualmente como 
uma das alternativas de cuidados não familiares em razão das mudanças com contexto do envelhecimento da população. No Brasil, mais de $65 \%$ das ILPIs são filantrópicas, por não disporem de recursos financeiros que permita oferecer um ambiente com mais condição, que muitas vezes diminui as chances de o idoso exercer o autogoverno e o poder de decisão, portanto o controle (MARTINS AA, et al., 2016).

Sabe-se que, a institucionalização altera a vida da pessoa idosa, uma vez que, passará a viver em um ambiente alheio à sua casa, dividir espaço com outras pessoas até então desconhecidas. Submeter-se-á também a regras, rotinas e cuidadores desconhecidos.

Essas mudanças podem acarretar insegurança, medo, ansiedade e outros impactos psicossociais (VECCHIA RD, 2015). No entanto, estudos constatam aspectos positivos nas ILPIs mediante atuação de bons profissionais da área da saúde, cuidadores e demais pessoas que trabalham com esse público e que realizam atividades sociais, culturais e de lazer na busca de proporcionar um ambiente prazeroso e motivador para os idosos (SOUZA RTJ, et al., 2016).

Faz-se necessário a elaboração de novos programas e ações voltadas para o idoso institucionalizado, levando em consideração a capacitação dos profissionais empenhados nessa tarefa, a sensibilidade da sociedade, apoio do poder público com um objetivo comum, proporcionar qualidade de vida a essa esfera da população.

Pesquisas têm demonstrado a necessidade de um novo modelo de cuidado e que, colabore com todo o pensamento direcionado a esse público sob o olhar da nova situação epidemiológica e demográfica (TOLDRÁ CR, et al., 2014).

É com base em todo este leque de informações acerca do envelhecimento que o os profissionais da saúde, como integrante da equipe do cuidado, deve conhecer a realidade das famílias nos aspectos mentais, sociais e demográficos, proporcionado assistência integral ao idoso e a família, estabelecendo metas e elaborando um plano assistencial com base nas necessidades fisiopatológicas da população idosa.

Além do mais, deve prestar assistência e capacitação aos cuidadores para participarem ativamente do cuidado prestado ao idoso, na tentativa de evitar algum tipo de agressão por parte da população estudada (BRASIL, 2014).

Assim, analisar as condições de vida da pessoa idosa, torna-se fundamental para a elaboração e desenvolvimento de alternativa de intervenção em programas de saúde, e políticos sociais. Sugere-se também a realização de mais estudos e práticas de saúde, na busca de possibilitar uma qualidade de vida no processo de envelhecimento (BARDIN L, 2016).

Com vistas nesse contexto em que a qualidade de vida do idoso institucionalizado passa a ser tratada como prioridade utilizou-se o presente estudo com o intuito de instigar a participação da sociedade nessa causa tão importante e presente aos nossos olhos cotidianamente. Deste modo, o presente estudo teve como objetivo avaliar os aspectos relacionados com a qualidade de vida dos idosos institucionalizados na cidade do Crato no estado do Ceará.

\section{MÉTODOS}

Trata-se de uma pesquisa descritiva, exploratória, e com abordagem qualitativa que busca o entendimento da realidade humana vivenciada pelos idosos institucionalizados no que concerne à qualidade de vida. $O$ estudo foi realizado em uma ILPI de caráter filantrópico de uma cidade do interior do estado do Ceará, mas, precisamente na região Sul do Ceará.

Como critério de inclusão optou-se pelas participantes com idade de 70 e mais de 80 anos e com capacidade cognitiva para responderem as indagações solicitadas. E optou-se por excluir os questionários respondidos de forma incompleta e que por algum motivo não estivessem de acordo com os objetivos do estudo. Os dados foram coletados no período de setembro a novembro de 2017 após assinatura do TCLE (Termo de Consentimento Livre e Esclarecido).

As entrevistas e todo o material obtido foram transcritos na íntegra, buscando facilitar a captação de todos os detalhes, como pausas e entonações, para uma maior aproximação com o objeto de estudo abordou-se 
as características referentes: as alterações morfológicas dos idosos, quanto as práticas de lazer na instituição, sobre os profissionais que prestam assistência os idosos e quanto a percepção dos mesmos acerca da qualidade de vida na ILPI.

No que concerne à análise dos dados obtidos com as entrevistadas estão demostrados na forma de tabela (sócio demográfico) e as respostas apresentadas foram transcritas e analisadas aparte do método de Bardin. Esse método é utilizado como um mecanismo para a compreensão da construção de significados que os atores sociais, exteriorizam nas falas e, também, caracteriza-se por ser um conjunto de procedimentos utilizados para a análise dos resultados coletados.

A análise temática desmembra-se em três fases de suma importância: 1) Pré-análise; 2) Exploração do material utilizado e tratamento dos resultados alcançados e 3) Interpretação (MOURA DMM e VERAS PP, 2017).

O estudo respeitou todas as recomendações formais advindas da resolução no 466/12 e sua complementação a Lei 510/16 do Conselho Nacional de Saúde/ Ministério da Saúde, referente a estudos com seres humanos (BRASIL, 2013).

Esta resolução incorpora sob a ótica do indivíduo e da coletividade, as quatro referências básicas da Bioética: autonomia, justiça, beneficência, não maleficência entre outros, garantindo os direitos e deveres do estado, da comunidade científica e dos sujeitos da pesquisa. Onde o projeto para esse estudo foi submetido à Plataforma Brasil e aprovado mediante parecer no 2.231.886 em 22 de agosto de 2017.

\section{RESULTADOS E DISCUSSÃO}

A grande demanda de idosos dentro de instituições de longa permanência leva a necessidade de análise dos fatores que podem contribuir para a baixa qualidade de vida. Foi realizada a entrevista as idosas que residem no Abrigo.

Para atender os objetivos propostos, inicialmente caracterizaram-se os sujeitos do estudo com informações de acordo com sua situação sócio-cultural-demográfica (Tabela 1). Participaram do estudo 13 (treze) idosos residentes na ILPI e todas do sexo feminino com idade entre 70 e mais de 80 anos e, o tempo de admissão na referida instituição variou de um a mais de trinta anos; seis com fundamental completo, quatro com ensino médio completo e duas com nível superior, onze são solteiras, duas casadas. Em se tratando da renda nove recebe um salário e quatro até dois salários mínimos. Todas residem na cidade onde foi realizada a pesquisa. 
Tabela 1 - Perfil sócio demográfico das idosas residentes em uma Instituição de Longa Permanência, em Crato - CE, 2017.

\begin{tabular}{|c|c|c|}
\hline Variáveis sócio demográficas & $\mathbf{N}$ & $\%$ \\
\hline \multicolumn{3}{|l|}{ Idade } \\
\hline Entre 70 e 80 anos & 4 & 30,7 \\
\hline Mais de 80 anos & 9 & 69,3 \\
\hline Total & 13 & 100 \\
\hline \multicolumn{3}{|l|}{ Estado civil } \\
\hline Solteira & 11 & 84,6 \\
\hline Casada & 2 & 15,4 \\
\hline Viúva & - & - \\
\hline Separada & - & - \\
\hline Total & 13 & 100 \\
\hline \multicolumn{3}{|l|}{ Escolaridade } \\
\hline Analfabeta & - & - \\
\hline Ensino fundamental incompleto & 6 & 46,3 \\
\hline Ensino fundamental completo & 1 & 7,6 \\
\hline Ensino médio incompleto & - & - \\
\hline Ensino médio completo & 4 & 30,7 \\
\hline Ensino superior incompleto & - & - \\
\hline Ensino superior completo & 2 & 15,4 \\
\hline Total & 13 & 100 \\
\hline \multicolumn{3}{|l|}{ Renda mensal } \\
\hline 1 salário mínimo* & 9 & 69,3 \\
\hline 2 salários mínimos & 4 & 30,7 \\
\hline Total & 13 & 100 \\
\hline \multicolumn{3}{|l|}{ Há quanto tempo reside na ilp? } \\
\hline 1 a 10 anos & 7 & 54 \\
\hline 10 a 20 anos & 2 & 15,4 \\
\hline 20 a 30 anos & 1 & 7,6 \\
\hline Mais de 30 anos & 2 & 15,4 \\
\hline Não sabem & 1 & 7,6 \\
\hline Total & 13 & 100 \\
\hline
\end{tabular}

Legenda: *Valor do salário mínimo na época da pesquisa: $\mathrm{R} \$$ 938,00. Fonte: Souza FJM, et al., 2020.

Nos países em desenvolvimento, o processo de envelhecimento ocorre de forma mais lenta, no entanto, no Brasil esse processo ocorreu de forma rápida e desvinculada de políticas sociais efetivas na atenção à saúde da pessoa idosa, o que de certa forma compromete a qualidade de vida dos mesmos.

Isso pode estar relacionado às mudanças de estilo de vida que estão sendo adotas pelos homens ao procurarem mais os serviços de saúde e as novas políticas voltadas ao seu atendimento, porém, é sabido que a mortalidade por sexo é maior para os homens quando comparados com as mulheres devido uma maior exposição a fatores de risco como o álcool, o tabaco e as doenças cardiovasculares e as mulheres ainda apresentam maior longevidade de certa forma por cuidarem mais de sua saúde (RAMOS et al., 2013).

Divergindo dos achados de Ra NL, (2009), que verificaram em seu estudo que os idosos institucionalizados residentes na zona urbana geralmente possuem companheiro e sem nível de instrução, e idade entre 60 e 70 anos, neste estudo a maioria dos sujeitos é solteira, possuem certo grau de escolaridade e em sua maioria tem mais de 80 anos de idade. Porém, corrobora com os autores quando se analisa a renda mensal que é predominantemente de um salário mínimo (VERAS PR e OLIVEIRA M, 2018).

Com o intuito de facilitar a análise dos dados da pesquisa, as falas que apresentavam maior relevância para a temática proposta foram organizadas em quatro categorias: 1) alterações morfofuncionais; 2) participação em eventos de lazer; 3) assistência profissional; e 4) qualidade de vida na ILPI. Com o intuito de manter o anonimato, os idosos tiveram pseudônimos escolhidos entre os nomes de cores. 


\section{Categoria 1 - Alterações morfofuncionais.}

Ao iniciar a entrevista, as idosas foram indagadas sobre as mudanças no organismo provocadas pelo envelhecimento e, surgiram as seguintes respostas:

"Sinto dificuldade na vista, às vezes nos pés, sinto as pernas doer" (Azul).

"Diminuição para o trabalho e, também, e a diminuição da vista, perda de forças, escuto bem" (Verde).

"Não aceitar e, não poder fazer tudo o que quer fazer, não tenho mais disposição" (Marrom).

As alterações anatômicas são consideradas pontos importantes para determinação da qualidade de vida. Conforme Fuhrmann AC, et al. (2015), o declínio funcional relacionado a presença de algumas patologias crônicas leva a um determinado grau de dependência para a realização de atividades complexas ou mesmos as mais simples.

Vale ressaltar que o envelhecimento traz desconfortos para os idosos quando se percebem incapazes de realizar as suas atividades do cotidiano. Contudo, essa condição não se apresenta necessariamente como pessimismo, mas apenas a aceitação de uma condição que as idosas não mais se vêm com capacidades para mudanças.

O declínio funcional associado com algumas patologias crônicas conduz o idoso a não realização das atividades diárias, das mais simples às complexas, tornando-o dependente de outras pessoas (DERHUN FM, et al., 2019).

Entende-se que o envelhecimento por si só já limita o idoso para a realização de atividades que até então realizavam. Assim, faz-se necessário um novo olhar para a pessoa em fase de envelhecimento no sentido de repensar a política pública voltada para esse público, reelaborando e elaborando medidas para prevenção e reabilitação de determinadas limitações advindas com a idade e consequentemente proporcionar melhor qualidade de vida (PIMENTEL WRT, et al., 2015).

Alguns idosos mesmo diante das limitações, ainda realizam certas atividades mais simples outros dependem da ajuda de alguém em razão de doenças crônico degenerativas, impedindo a manutenção da autonomia e independência.

Nota-se que a realização de tarefas por mais simples que sejam, podem impactar de forma positiva para a manutenção do aporte estrutural e funcionamento do organismo, tornando os idosos mais autônomos, confiantes e responsáveis pelos os seus atos (CABRAL JR, et al., 2015).

\section{Categoria 2 - Participação em eventos de lazer.}

No que tange a participação em atividades de lazer, as idosas institucionalizadas, mencionaram respostas positivas. Vejamos:

"Sim, duas vezes por ano e esse passeio é feito com todas as idosas" (Verde).

"Vou para os lazeres sempre que tem na chácara e piscina" (Cinza).

"É ótimo porque estou fazendo uma higiene mental e tô ocupando o tempo e por minha idade me sinto feliz em fazer alguma coisa e tem muitas orações também é isso ai" (Laranja).

As atividades de lazer são consideradas pelas pesquisadas como ambientes de descontração, onde podem desfrutar das alegrias, usufruir do convívio social com outras pessoas, repousar com tranquilidade, jogar conversa fora e sentirem-se por alguns momentos fora da ILPI. Vale ressaltar que é direito do idoso participar de diversas atividades como, por exemplo: esportivas, artísticas, culturais, lazer, já mencionada anteriormente (TAVARES SMD, et al., 2016).

As atividades de lazer têm sido consideradas nos últimos anos como formas de ocupação dos tempos livres do homem. São espaços na vida onde as pessoas como defende Ferreira CFG e Nascimento AR (2015), 
podem desfrutar prazeres, tranquilidade e até descanso. Os idoso tem como direito benefícios nas atividades culturais, artísticas, esportivas e de lazer. O Sistema Único de Saúde (SUS) deve garantir atenção integral a população idosa, com ênfase no envelhecimento saudável e ativo e com vínculo familiar (BRASIL, 2016).

É importante reforçar que a prática de lazer quando associadas a outras atividades torna-se de fundamental importância para a qualidade de vida e no modo como as outras práticas podem contribuir na vida dos idosos, especialmente os institucionalizados, prevenindo doenças, como a depressão, evita o isolamento e promove o convívio social (MARUO TL, et al., 2016).

O convívio social pode possibilitar benefícios relacionados a fatores físicos, cognitivos e funcionais da pessoa idosa mediante a troca de experiências, melhorando a autoestima e a sensação de pertencimento da sociedade. Assim, engajar o idoso em momentos de alegria na comunidade, é importante para a qualidade de vida. A estimulação cognitiva em idosos por meio da leitura favorece uma saúde mental e social com mais qualidade, diminuindo diversas doenças como a depressão (CAMPOS VCA, et al., 2014).

\section{Categoria 3 - Assistência profissional.}

No que tange a assistência profissional na ILPI para com as idosas, as respostas foram expressivas:

"Bem, quando eu preciso das meninas que trabalham aqui graças a Deus elas me atendem muito bem, botam o colírio no meu olho, faz limpeza no meu quarto. Está ótimo" (Laranja).

"São boas, atenciosas, responsável e quando a gente pede alguma coisa a elas sempre é atendida com muito carinho" (Preto).

"Os profissionais são pessoas excelentes, são preparada, fizeram curso de cuidador de idosos" (Verde).

As falas das pesquisadas deixam transparecer a maneira respeitosa como são tratadas na instituição onde o amor, a compreensão e a valorização se fazem presentes no cotidiano da ILPI. Ressalta-se que os idosos institucionalizados despertam uma maior preocupação em razão da fragilidade social, psicológica e física que podem apresentar. Assim, cuidados especiais são necessários, justificados pelo grau de dependência, presenças de determinadas doenças e outros tipos de complicações decorrentes do envelhecimento (FREITAS MG, et al., 2015).

De acordo com os relatos, as medidas tomadas dentro da instituição são adequadas para atender as suas necessidades. Isso não exclui a necessidade de uma melhor assistência que pode ocorrer por meio de uma equipe multiprofissional para determinar uma melhor assistência e promoção da saúde (CHRISTOFANI LR, 2013). O paciente conforme Duarte GP e Figueiredo LRU (2014), busca na instituição de saúde um tratamento mais humano, atencioso e respeitoso, o conforto emocional e a preservação da sua vida. Respeitar o próximo requer um caráter profundamente afável. Neste sentido, a prática profissional qualificada e humanizada tornase mandatória para viabilizar a efetivação da qualidade de vida desses sujeitos.

Conforme Del Duca GF, et al. (2012), a qualificação continuada e um ambiente de estudos, reuniões e supervisão devem ser assegurados para os profissionais, onde estes possam compartilhar dúvidas, angústia e anseios, assim, o resultado será um atendimento de qualidade. Nem sempre será possível ter uma equipe completa, mas, a Instituição de Longa Permanência para Idosos deve manter princípios normativos das ações de seu pessoal, como tratar os idosos com dignidade e respeito, permitir guarda e uso de objetos pessoais, possibilitar liberdade na interação social, respeitar a prática religiosa individual e favorecer a privacidade (PEREIRA SD, et al., 2015).

\section{Categoria 4 - Qualidade de vida na ILPI.}

Com relação à qualidade de vida na instituição, as pesquisadas foram enfáticas nas suas proposições:

"Está sendo boa. O pessoal é bom comigo, não me falta nada e não fala de me botar pra fora" (Azul).

"Bem, aqui tá bem porque é uma casa de repouso, aí eu me sinto bem [...] pela idade que tenho é bom ficar aqui mesmo, só isso" (Laranja). 


\begin{abstract}
"É muito boa. Hoje eu não vou mais a rua. Quando cheguei aqui só vivia indo a rua, agora sinto muita dor nas pernas, tenho boa alimentação" (Preto).

"Ótima, a qualidade de vida tá maravilhosa porque eu gosto daqui e o povo coopera comigo" (Bege).
\end{abstract}

Várias são as definições sobre qualidade de vida. Não há um consenso por parte dos estudiosos do assunto abordado o que dificulta um pensamento único. O que se entende é que fatores como saúde, relações familiares, atividades de lazer, trabalho, bem estar psíquico e social, também definem qualidade de vida. A qualidade de vida assume um caráter subjetivo quando, relaciona-se com a maneira como o sujeito se percebe no contexto social. Assim, as pesquisadas institucionalizadas avaliam a qualidade de vida a partir da acomodação que lhes proporcionam um local seguro com boa alimentação e bom convívio social (MATIAS AGC, et al., 2015).

Um dos princípios de qualidade vida é apreciar, é valorizar parâmetros mais amplos do que apenas o controle de sintomas físicos ou psicológicos, a redução da mortalidade ou avanço da expectativa de vida. Qualidade de vida está relacionada a um dos anseios básicos do ser humano, que é viver bem e sentir-se bem. Diferentes fatores levam a permanência e muitas vezes a melhora desta qualidade, entre elas os hábitos de vida, o conviveu social e familiar, as práticas em grupos sendo elas lúdicas ou recreativas, entre outras, tais fatores são determinante para uma melhora qualidade de vida do idoso (MARIN MJS, et al., 2012).

A percepção de qualidade de vida depende de fatores culturais do próprio indivíduo, assim como da sociedade a qual está inserido. A satisfação dos diferentes domínios que fazem parte da qualidade de vida são essenciais para determinação de uma melhor exploração desse conceito. Determinadas mudanças na qualidade de vida que muitas vezes são orientados por profissionais de saúde podem contribuir de maneira importante para o aumento do grau de qualidade de vida experimentado pelo idoso nas ILPI (CONFORTIN CS, et al., 2016).

Quando o idoso vai morar em uma instituição, tem que enfrentar o momento de sair de sua casa, deixar seus pertences e familiares para se adaptar à nova realidade, a qual muitas vezes não oferece condições favoráveis para uma vida digna (VERAS PR e OLIVEIRA M, 2018). Segundo Setien JÁ (2014), a instituição que recebe o idoso deve manter o contato com a família, fugindo da tendência de transformá-lo em vítima e os familiares em vilões, compreendendo a dinâmica da situação, isso favorece ao idoso uma melhor qualidade de vida durante o período de permanência na Instituição de Longa Permanência.

\title{
CONCLUSÃO
}

Mediante os resultados da pesquisa percebeu-se que os idosos têm a instituição de longa permanência para a pessoa Idosa (ILPI) um local de segurança e que lhes proporcionam momentos de descontração, alegrias e convívio social. São tratadas com amor, compreensão e valorização e dispõem de uma boa alimentação. Espera-se com este estudo contribuir com todos os envolvidos, com esta parcela da população no sentido de repensar valores e buscar mais conhecimentos sobre a temática que proporcione uma melhor atuação nos diferentes espaços. A escassez de pesquisas atuais sobre o tema dificulta uma análise mais expressiva sobre o assunto. Sugere-se a realização de mais estudos nesse sentido, que venha possibilitar uma investigação mais profunda sobre a realidade social dos idosos institucionalizados.

\section{REFERÊNCIAS}

1. ABREU JF, et al. Qualidade de vida e fatores associados em idosos de um Centro de Referência à Pessoa Idosa. Revista Kairós Gerontologia, 2017; 2(2): 333-352.

2. BARDIN L. Análise de Conteúdo. São Paulo, 2016; 167p.

3. CAMPOS VCA, et al. Qualidade de vida de idosos praticantes de atividade física no contexto da estratégia da família. Texto e Contexto Enfermagem. Florianópolis, 2014; 23(4): 889-897.

4. BRASIL. Conselho Nacional de Saúde. Resolução $n^{\circ} 466,2012$. Diretrizes e Normas regulamentadoras de pesquisa envolvendo seres humanos. Brasília, 13 jun. Seção 1, p. 59, 2013.

5. BRASIL. Ministério da Saúde. Secretaria de Atenção à Saúde. Departamento de Atenção Básica. Caderno de Atenção Básica - Prevenção clínica de doenças cardiovasculares, cerebrovasculares e renais. Brasília, 2014. 
6. BRASIL. Ministério da Saúde. Caderneta de Saúde da Pessoa Idosa: Manual de Preenchimento. Brasília: Ministério da Saúde, 2016.

7. CABRAL JR, et al. Oficinas de educação em saúde em idosos: uma estratégia de promoção da qualidade de vida. Revista Enfermagem Digital Cuidado e Promoção da Saúde, 2015; 1(2): 62-69.

8. CHRISTOFANI LR. Qualidade de vida para a terceira idade. Rio de Janeiro, 2013; 11(4).

9. CONFORTIN CS, et al. Atividades no lazer e Qualidade de vida de Idosos de um Programa de Extensão Universitária em Florianópolis (SC). Bras Ativ Fis Saúde, 2016; 19(4): 494-503.

10. DEL DUCA GF, et al. Indicadores da institucionalização de idosos: estudo de casos e controles. Rev Saúde Pública, 2012; 46(1): 147-153.

11. DERHUN FM, et al. O Centro de Convivência para idosos e sua importância no suporte à Família e a Rede de Atenção à Saúde. Escola Anna Nery, 2019; 23(2).

12. DUARTE GP, FIGUEIREDO LRU. A vida cotidiana e a qualidade de vida de pacientes atendidos na atenção primária de saúde. Cadernos de Terapia Ocupacional da UFSCar, São Carlos, 2014; 18(1): 34-47.

13. FERREIRA CFG, NASCIMENTO AR. Qualidade de vida e autoestima de idosos na comunidade. Ciência \& Saúde Coletiva, 2015; 13(4): 123-132.

14. FREITAS MG, et al. Idosos atendidos em serviços de urgência no brasil: um estudo para vítimas de quedas e de acidentes de transito. Ciência \& Saúde Coletiva, Rio de Janeiro, 2015; 20(3): 701-712.

15. FUHRMANN AC, et al. Associação entre a capacidade funcional de idosos dependentes e a sobrecarga do cuidador familiar. Revista Gaúcha de Enfermagem, Porto Alegre, 2015; 36(1): 14-20.

16. KHAURY HTT, NEVES ACS. Percepção de Controle e Qualidade de Vida entre Idosos Institucionalizados e não Institucionalizados. Rev. Bras. Geriatr. Gerontol, 2014; 17(3): 553-565.

17. KLEIN LH, LODOVICI FMM. A moradia na Velhice: A Família, a instituição asilar, as redes de apoio, a cobertura da mídia. ANAIS: Semana de Gerontologia e Simpósio Internacional de Gerontologia Social. Programa de Estudos de Pós- graduação em Gerontologia da PUC- SP, 2015.

18. MARIN MJS et al. Percepção de idosos hipertensos sobre suas necessidades de saúde. Rev. Esc. Enferm, São Paulo, 2012; 46(1) 103-110.

19. MAROU TL. Estratégia Saúde da Família e a atenção ao idoso: experiências em três municípios brasileiros. Cad. Saúde Pública, Rio de Janeiro, 2016; 27(4): 779-786.

20. MARTINS AA, et al. Conhecendo o perfil clínico do idoso institucionalizado: Um Olhar sobre a qualidade da assistência. Revista Tendência da Enfermagem Profissional, 2016; 9(2): 2176-2181.

21. MATIAS AGC, et al. Análise fatorial de sintomas depressivos e ocorrência de quedas em idosos. Scientia Medica, $2015 ; 25(1): 2-8$.

22. MOURA DMM, VERAS PP. Acompanhamento e envelhecimento humano em Centro de Convivência. Physis Revista de saúde coletiva, Rio de Janeiro, 2017; 27(1): 19-39.

23. OLIVEIRA TC, et al. Diferenciais sócio-demográficos da mortalidade de idosos em idades precoces e longevas. Revista Baiana de Saúde Pública, 2015; 39(2): 249-261.

24. Organização Mundial da Saúde. Diminuindo diferenças: a prática das políticas sobre determinantes sociais da saúde: documento de discussão. Rio de Janeiro: OMS; 2016.

25. PEREIRA SD, et al. Qualidade de Vida e Situação de idosos: um estudo de base populacional no sertão central do Ceará. Revista Brasileira Geriatria Gerontologia. Rio de Janeiro, 2015; 18(4): 893-898.

26. PIMENTEL WRT, et al. Quedas e qualidade de vida: associação com aspectos emocionais em idosos comunitários. Geriatria e Gerontologia Aging, 2015; 9(2): 42-48.

27. PIMENTEL WRT, et al. Quedas e qualidade de vida: associação com aspectos emocionais em idosos comunitários. Geriatrics Gerontology and Aging, 2015; 9(2): 42-48.

28. RA NL. Envelhecendo com qualidade de vida. Programa Gerontologia. Porto Alegre, RS. 2009.

29. RAMOS LR, et al. Fatores determinantes do envelhecimento saudável em idosos residentes em centro urbano: Projeto Epidoso, São Paulo. Caderno de Saúde Pública, Rio de Janeiro, 2013; 19(3): 793-798.

30. SANTOS FH, et al. Envelhecimento: um processo multifatorial. Psicologia em Estudo. Maringá, 2014; 14(1): 3-10, 2014.

31. SETIEN JA. Abordagem do Idoso em Programas de Saúde da Família. Cad. Saúde Pública, Rio de Janeiro, 2014; 19(3): 839-847.

32. SOUZA RTJ, et al. Envelhecer no Brasil: a construção de um modelo de cuidado no estudo do Pará. Cad. Saúde Pública, 2016; 13(8): 245-256.

33. TAVARES SMD, et al. Qualidade de autoestima de idosos na comunidade. Revista Ciência e Saúde Coletiva, 2016; 21(11): 3557-3564.

34. TOLDRÁ CR, et al. Promoção da Saúde e da qualidade de vida com idosos por meio de práticas corporais. O Mundo da Saúde. São Paulo, 2014; 38(2): 159-169.

35. VECCHIA RD. Qualidade de vida na terceira idade: um conceito subjetivo. Rev Bras Epidemiol, 2015; 8(3): $246-252$.

36. VERAS RP, OLIVEIRA M. Envelhecer no Brasil: a construção de um modelo de cuidado. Ciência e Saúde Coletiva, 2018; 23(6): 89-101. 